\title{
The authorship of the taxa in the paper of Lienig \& Zeller, 1846
}

\author{
REINHARD GAEDIKE ${ }^{1}$ \\ 1 Florusstraße 5, 53225 Bonn, Germany; tinagma@msn.com \\ http://zoobank.org/1258F9AB-85AD-434F-8B1C-87D93971D3C8
}

Received 10 April 2014; accepted 3 June 2014; published: 8 September 2014

Subject Editor: Lauri Kaila.

\begin{abstract}
The authorships of the taxa described in the paper of Lienig \& Zeller, 1846, are discussed. The authorship of all species newly described in this paper should be assigned to both. A list of the taxa newly described in that paper and their current taxonomic status is provided.

Zusammenfassung. Die Autorenschaft für die in der Arbeit von Lienig \& Zeller, 1846, beschriebenen Taxa wird diskutiert. Für alle in dieser Arbeit neu beschriebenen Arten sollten beide Namen als Autoren angesehen werden. Eine Liste der neubeschriebenen Taxa mit ihrem gegenwärtigen taxonomischen Status wird vorgelegt.
\end{abstract}

\section{Introduction}

In a paper on the Latvian regions of Livland and Curland (Vidzeme and Kurzeme) by Lienig and Zeller (1846) a number of species from several Lepidoptera families were newly described; however, there exist conflicting views with regard to their correct authorships. The reasons for such uncertainty lie in the ambiguous way in which the authorship of this paper is expressed: “...Bearbeitet von Friederike Lienig, geb. Berg, mit Anmerkungen von P. C. Zeller. [worked by Friederike Lienig ..., with annotations by P. C. Zeller]" and in the fact that the names of the described taxa were credited to a variety of authors: "Lienig nov. sp.;" "Zell. nov. sp.;" "nov. spec.;" "Tischer in litt.;" "Heyden nov. sp.;" "Tr[eitschke]. in litt." As a result, the new taxa were subsequently attributed variously to "Lienig", "Zeller" or "Lienig \& Zeller".

Examining the text it is hardly possible to be certain in each case which part of an original description was written by Lienig and which by Zeller. Most, if not all, descriptions contain elements contributed by both authors (e.g. biological information and descriptions of the larva are usually by Lienig), and the use of the personal pronoun $I$ refers in some instances to Lienig in others to Zeller. It seems therefore justified uniformly to attribute the authorship of all the taxa described as new in that paper jointly to "Lienig \& Zeller". 


\title{
List of the taxa newly described by Lienig and Zeller 1846, with their original attribution and their current taxonomic status
}

\author{
acuminatana Zell. nov. sp. (Grapholitha[sic!])(p. 256)
}

Current status: Dichrorampha acuminatana (Lienig \& Zeller, 1846) - Tortricidae. arctata Lienig nov. sp. (Cidaria) (p. 199)

Current status: Martania taeniata (Stephens, 1831)

Syn.: Cidaria arctata Lienig \& Zeller, 1846 - Geometridae.

bisontella Lienig nov. sp. (Ochsenheimeria) (p. 274)

Current status: Ochsenheimeria urella Fischer v. Röslerstamm, 1842

Syn.: Ochsenheimeria bisontella Lienig \& Zeller, 1846 - Ochsenheimeriidae.

ciniflonella Lienig nov. sp. (Depressaria) (p. 280)

Current status: Exaeretia ciniflonella (Lienig \& Zeller, 1846) - Depressariidae. comariana Lienig nov. sp. (Teras) (p. 263)

Current status: Acleris comariana (Lienig \& Zeller, 1846) - Tortricidae conterminata nov. spec. (Larentia) (p. 197)

Current status: Eupithecia conterminata (Lienig \& Zeller, 1846) - Geometridae costiguttella Lienig nov. sp. (Gelechia) (p. 290)

Current status: Brachmia dimidiella (Denis \& Schiffermüller, 1775)

Syn: Gelechia costiguttella Lienig \& Zeller, 1846

cuphana Tischer in litt. (Grapholitha[sic!]) (p. 245)

Current status: Rhopobota stagnana (Denis \& Schiffermüller, 1775)

Syn.: Grapholita cuphana Lienig \& Zeller, 1846. A junior primary homonym of Grapholita cuphana Duponchel, 1843 - Tortricidae, Olethreutinae.

Remarks. According to J. Baixeras (pers. comm.) this homonymy has remained hitherto unnoticed.

deauratella Lienig nov. sp. (Coleophora) (p. 295)

Current status: Coleophora deauratella Lienig \& Zeller, 1846 - Coleophoridae.

diaphanella Lg. nov. sp. (Gelechia) (p. 285)

Current status: Helcystogramma rufescens (Haworth, 1828)

Syn.: Gelechia diaphanella Lienig \& Zeller, 1846. - Gelechiidae.

flavaginella Lienig (Coleophora) (p. 295)

Current status: Coleophora sternipennella (Zetterstedt, 1839)

Syn: Coleophora flavigenella Lienig \& Zeller, 1846 - Coleophoridae.

fuliginosella Lienig nov. sp. (Tinea) (p. 273)

Current status: Elatobia fuliginosella (Lienig \& Zeller, 1846) - Tineidae.

gallicolana Heyden nov. sp. (Grapholitha) (p. 255)

Current status: Pammene gallicolana (Lienig \& Zeller, 1846) - Tortricidae.

gimmerthaliana Lienig nov. sp. (Grapholitha[sic!]) (p. 247)

Current status: Epinotia gimmerthaliana (Lienig \& Zeller, 1846) - Tortricidae.

Remarks: See gimmerthaliania.

gimmerthaliania Lienig nov. sp. (Grapholitha[sic!]) (p. 247)

Current status: Epinotia gimmerthaliana (Lienig \& Zeller, 1846) - Tortricidae. 
Remarks: The name gimmerthaliania is an incorrect (of a multiple) original spelling. Although it is used in the main heading of this species, the spelling gimmerthaliana commences the Latin diagnosis and was subsequently accepted as the correct spelling. grandaevana Lienig nov. sp. (Pedisca) (p. 238)

Current status: Epiblema grandaevana (Lienig \& Zeller, 1846) - Tortricidae.

hepatariella Lienig nov. sp. (Depressaria) (p. 282)

Current status: Levipalpus hepatariella (Lienig \& Zeller, 1846) - Depressariidae.

hübneriana [sic!] Lienig nov. sp. (Paedisca) (p. 237)

Current status: Pelochrista huebneriana (Lienig \& Zeller, 1846) - Tortricidae.

immundata nov. spec. (Larentia) (p. 194)

Current status: Eupithecia immundata (Lienig \& Zeller, 1846) - Geometridae.

improbata Tr. in litt. (Larentia) (p. 196)

Current status: Gymnoscelis rufifasciata (Haworth, 1809)

Syn: Larentia improbata Lienig \& Zeller, 1846 - Geometridae.

incana Zell. nov. sp. (Grapholitha[sic!]) (p. 239)

Current status: Eucosma wimmerana (Treitschke, 1835)

Syn: Grapholitha[sic!] incana Lienig \& Zeller, 1846 - Tortricidae.

inquinatalis Lienig nov. sp. (Scopula) (p. 205)

Current status: Udea inquinatalis (Lienig \& Zeller, 1846) - Crambidae.

internella Lienig nov. sp. (Gelechia) (p. 291)

Current status: Thiotricha subocellea (Stephens, 1834)

Syn: Gelechia internella Lienig \& Zeller, 1846 - Gelechiidae.

lamana Zell. nov. sp. (Phoxopteris) (p. 257)

Current status: Bactra furfurana (Haworth, 1811)

Syn: Phoxopteris lamana Lienig \& Zeller, 1846 - Tortricidae.

leguminana Lienig nov. sp. (Grapholitha[sic!]) (p. 253)

Current status: Cydia leguminana (Lienig \& Zeller, 1846) - Tortricidae.

lienigiana $\mathrm{v}$. Tischer in litt. (Penthina) (p. 213)

Current status: Argyroploce lediana Linnaeus, 1758 - Tortricidae.

Syn: Penthina lienigiana Lienig \& Zeller, 1846

lienigiella Zell. n. sp. (Cosmopteryx) (p. 298)

Current status: Cosmopterix lienigiella (Lienig \& Zeller, 1846) - Cosmopterigidae.

lucivagana Lienig nov. sp. (Sericoris) (p. 229)

Current status: Celypha rurestrana (Duponchel, 1843)

Syn: Sericoris lucivagana Lienig \& Zeller, 1846 - Tortricidae.

nitidulana Lienig nov. sp. (Phoxopteris) (p. 259)

Current status: Gypsonoma nitidulana (Lienig \& Zeller, 1846) - Tortricidae.

obumbratana Lienig nov. sp. (Grapholitha[sic!]) (p. 240)

Current status: Eucosma obumbratana (Lienig \& Zeller, 1846) - Tortricidae.

ochsenheimeriana Lienig nov. sp. (Grapholitha[sic!]) (p. 249)

Current status: Pammene ochsenheimeriana (Lienig \& Zeller, 1846) - Tortricidae.

padana Lienig nov. sp. (Grapholitha[sic!]) (p. 243)

Current status: Epinotia signatana (Douglas, 1845) - Tortricidae.

Syn: Grapholitha[sic!] padana (Lienig \& Zeller, 1846) 
pallifrontana Zell. In litt. (Grapholitha[sic!]) (p. 251)

Current status: Grapholita pallifrontana Zeller, 1845

Syn: Grapholitha[sic!] pallifrontana Lienig \& Zeller, 1846 - Tortricidae.

Remarks: Zeller (1845) named on p. 14 under Grapholitha[sic!] "1630 Pallifrontana Zeller:

Kreisewitz, im Sommer einzeln" and figured (fig. 15) this species as Graph. pallifrontana in:

5. Bericht des schlesischen Tauschvereins für Schmetterlinge 1844. This figure is the valid description of the taxon.

palustrana Lienig nov. sp. (Sericoris) (p. 230)

Current status: Phiaris palustrana (Lienig \& Zeller, 1846) - Tortricidae.

pascualis Lienig nov. sp. (Botys) (p. 206)

Current status: Udea lutealis (Hübner, 1809)

Syn: Botys pascualis Lienig \& Zeller, 1846 - Crambidae.

pinicolana Zell. nov. sp. (Grapholitha[sic!]) (p. 242)

Current status: Zeiraphera griseana (Hübner, 1799)

Syn: Grapholitha[sic!] pinicolana Lienig \& Zeller, 1846 - Tortricidae.

pinivorana Zell. nov. sp. (Coccyx) (p. 225)

Current status: Rhyacionia pinivorana (Lienig \& Zeller, 1846) - Tortricidae.

plumbatana Zell. nov. spec. (Grapholitha[sic!]) (p. 249)

Current status: Pammene splendidulana (Guenée, 1845)

Syn: Grapholitha[sic!] plumbatana Lienig \& Zeller, 1846 - Tortricidae.

postremana Lienig nov. sp. (Sericoris) (p. 231)

Current status: Pristerognatha penthinana (Guenée, 1845)

Syn: Sericoris postremana Lienig \& Zeller, 1846 - Tortricidae.

prolongata Lienig nov. sp. (Larentia) (p. 198)

Current status: Eupithecia extensaria (Freyer, 1844)

Syn: Larentia prolongata Lienig \& Zeller, 1846 - Geometridae.

pruinosella Lienig nov. sp. (Gelechia) (p. 288)

Current status: Athrips pruinosella (Lienig \& Zeller, 1846) - Gelechiidae.

pulchella Lienig nov. sp. (Argyresthia) (p. 293)

Current status: Argyresthia pulchella Lienig \& Zeller, 1846 - Argyresthiidae.

redimitella Z. in litt. (Tin. Lampronia) (p. 271)

Current status: Lampronia redimitella (Lienig \& Zeller, 1846) - Prodoxidae. ruptana Lienig (Teras) (p. 261)

Current status: Acleris sparsana (Denis \& Schiffermüller, 1775)

Syn: Teras sparsana var. ruptana Lienig \& Zeller, 1846 - Tortricidae.

serraria Lienig nov. sp. (Cidaria) (p. 200)

Current status: Heterothera serraria (Lienig \& Zeller, 1846) - Geometridae.

splendidella Lienig nov. sp. (Coleophora) (p. 296)

Current status: Metriotes lutarea (Haworth, 1828)

Syn: Coleophora splendidella Lienig \& Zeller, 1846 - Coleophoridae.

strigulatella Lienig in litt. (Lithocolletis) (p. 300)

Current status: Phyllonorycter strigulatella (Lienig \& Zeller, 1846) - Gracillariidae. suspectana Lienig nov. sp. (Grapholitha) (p. 255)

Current status: Pammene suspectana (Lienig \& Zeller, 1846) - Tortricidae. 
temerella Lienig nov. sp. (Gelechia) (p. 284)

Current status: Anacampsis temerella (Lienig \& Zeller, 1846) - Gelechiidae.

torquatella Lienig nov. sp. (Oecophora) (p. 279)

Current status: Atemelia torquatella (Lienig \& Zeller, 1846) - Praydidae.

vacciniana Tischer in litt. (Grapholitha[sic!]) (p. 248)

Current status: Rhopobota myrtillana (Humphreys \& Westwood, 1845)

Syn: Grapholitha[sic!] vacciniana Lienig \& Zeller, 1846 - Tortricidae.

vacciniella Lienig nov. sp. (Epischnia)

Current status: Ortholepis vacciniella (Lienig \& Zeller, 1846) - Pyralidae.

vinctaria Lienig nov. spec. (Idaea) (p. 203)

Current status: Macaria loricaria (Eversmann, 1837)

Syn: Idaea vinctaria Lienig \& Zeller, 1846 - Geometridae.

\section{Acknowlegements}

I thank Klaus Sattler (Natural History Museum London) for useful comments and for linguistic corrections and Joaquin Baixeras for information concerning the taxonomic status of cuphana Lienig \& Zeller, 1846.

\section{References}

Zeller PC (1845) In: Fünfter Bericht des schlesischen Tauschvereins für Schmetterlinge, 1844: 1-19. [figs 1-16]

Lienig F, Zeller PC (1846) Lepidopterologische Fauna von Lievland und Curland. Bearbeitet von Friederike Lienig, geb. Berg, mit Anmerkungen von P. C. Zeller. Isis oder Encyclopaedische Zeitung von Oken, Leipzig 39(3/4): 175-302. 